\title{
Massive gravity simplified: a quadratic action
}

\author{
Ali H. Chamseddine ${ }^{a, b, c, d}$ and Viatcheslav Mukhanov ${ }^{e, f}$ \\ ${ }^{a}$ American University of Beirut, Physics Department, \\ Beirut, Lebanon \\ ${ }^{b}$ I.H.E.S., \\ F-91440 Bures-sur-Yvette, France \\ ${ }^{c}$ Université de Tours, \\ Parc de Grandmont, 37200 Tours, France \\ ${ }^{d}$ LE STUDIUM, Loire Valley Institute for Advanced Studies, \\ Tours and Orleans, France \\ e Theoretical Physics, Ludwig Maxmillians University, \\ Theresienstr. 37, 80333 Munich, Germany \\ ${ }^{f}$ Department of Physics, New York University, \\ New York, NY 10003, U.S.A. \\ E-mail: achamseddine@gmail.com, mukhanov@physik.lmu.de
}

AbstRACT: We present a simplified formulation of massive gravity where the Higgs fields have quadratic kinetic term. This new formulation allows us to prove in a very explicit way that all massive gravity theories considered so far inevitably have Boulware-Deser ghost in non-trivial fluctuations of background metric.

Keywords: Classical Theories of Gravity, Space-Time Symmetries

ARXIV EPRINT: 1106.5868 
In a series of papers we have used the Higgs mechanism to give mass to the graviton [13]. The requirement of Poincare invariance imposes rather severe conditions on the possible Higgs fields. Namely, in diffeomorphism invariant theories we are constrained to use four scalar fields $\phi^{A}, A=0,1,2,3$ to play the role of Higgs fields. All proposed Poincare and diffeomorphism invariant theories of massive gravity (see refs. in recent review [4]) can be reduced to Higgs gravity where the massive term is built out of the following diffeomorphism invariant combinations of the scalar fields [1]

$$
\bar{h}^{A B}=g^{\mu \nu} \partial_{\mu} \phi^{A} \partial_{\nu} \phi^{B}-\eta^{A B} .
$$

When the scalar fields acquire vacuum expectation values, proportional to the space-time coordinates

$$
\left\langle\phi^{A}\right\rangle=x^{A}
$$

their perturbations

$$
\chi^{A}=\phi^{A}-x^{A},
$$

around this broken symmetry background induce extra massive metric degrees of freedom. When combined with two degrees of freedom of GR graviton (which also becomes massive due to interactions with the background) they constitute a massive graviton. A Poincare invariant massive graviton must have five degrees of freedom. However, four scalar fields in general have four degrees of freedom, one of which, namely $\chi^{0}$, inevitably describes a ghost. There is a unique choice of the action for scalar fields, to order $\bar{h}^{2}$, where the ghost does not propagate around Minkowski background. This is the well-known Fierz-Pauli action [5]

$$
S_{\phi}=\frac{m_{g}^{2}}{8} \int d^{4} x \sqrt{-g}\left[\bar{h}^{2}-\bar{h}_{B}^{A} \bar{h}_{A}^{B}+O\left(\bar{h}^{3}\right)\right],
$$

where we raise and lower indices with Minkowski metric $\eta^{A B}$. If we restrict ourselves to quadratic terms and substitute in this action

$$
\bar{h}^{A B}=h^{A B}+\partial^{A} \chi^{B}+\partial^{B} \chi^{A}+\partial^{C} \chi^{A} \partial_{C} \chi^{B}+h^{\mu \nu} \partial_{\mu} \chi^{A} \partial_{\nu} \chi^{B}
$$

then we immediately find that up to second order in $h^{2}, h \chi$ and $\chi^{2}$ the field $\chi^{0}$ is the Lagrange multiplier and hence has no propagator. However, in the next order there appears the term $h\left(\dot{\chi}^{0}\right)^{2}$ and the field $\chi^{0}$ starts to propagate on a background which deviates from Minkowski background. This is the well known nonlinear Boulware-Deser ghost [6]. Because one can always add to the Fierz-Pauli term the higher order in $\bar{h}$ terms, there was a hope that there is a unique (up to the total derivatives) action, which can be written as an infinite series in powers of $\bar{h}$, where BD ghost does not appear [7]. In [3] we have shown that even in this theory the nonlinear ghost arises, but only in the fourth order of perturbation theory. However, in $[7,8]$ it was claimed that using fields redefinitions this ghost could be moved to the next orders and eventually might be canceled.

In this paper we will show that contrary to these claims the nonlinear ghost is inevitable in any theory described by action (4) irrespective of its nonlinear extension. With this purpose we first present a new simplified reformulation of massive gravity, where the action proposed in [7], will depend only quadratically on $\phi^{A}$. 
Let us introduce the four vector fields $e_{A}^{\mu}$, which are constrained to satisfy

$$
g^{\mu \nu}=e_{A}^{\mu} e_{B}^{\nu} \eta^{A B}
$$

Next we define

$$
S_{A B}=e_{A}^{\mu} \partial_{\mu} \phi_{B}-\eta_{A B},
$$

which is constrained to be symmetric

$$
S_{A B}=S_{B A},
$$

implying

$$
e_{A}^{\mu} \partial_{\mu} \phi_{B}-e_{B}^{\mu} \partial_{\mu} \phi_{A}=0
$$

There are 16 constraints on the 16 fields $e_{A}^{\mu}$ which could be solved unambiguously to determine $e_{A}^{\mu}$ in terms of $g^{\mu \nu}$ and $\partial_{\mu} \phi_{A}$. This can only be done perturbatively, which in turn implies that $S_{A B}$ depends on $g^{\mu \nu}$ and $\partial_{\mu} \phi_{A}$ nonlinearly. Let us consider the following diffeomorphism invariant action

$$
S=-\frac{1}{2} \int d^{4} x \sqrt{-g} R+\frac{m_{g}^{2}}{8} \int d^{4} x \sqrt{-g}\left[S^{2}-S_{A B} S^{A B}+O\left(S^{3}\right)\right],
$$

where

$$
S=S_{A B} \eta^{A B} .
$$

The constraints (5) and (6) can be imposed by using the method of Lagrange multipliers

$$
\frac{1}{2} \int d^{4} x \sqrt{-g}\left(\tau_{\mu \nu}\left(g^{\mu \nu}-e_{A}^{\mu} e_{B}^{\nu} \eta^{A B}\right)+2 \lambda^{A B} S_{A B}\right),
$$

with

$$
\tau_{\mu \nu}=\tau_{\nu \mu}, \quad \lambda^{A B}=-\lambda^{B A}
$$

We now show that the class of actions (9) is equivalent to the class of actions (3). Consider the matrices

$$
S_{A B}^{\prime}=e_{A}^{\mu} \partial_{\mu} \phi_{B}=S_{A B}+\eta_{A B},
$$

and their product

$$
\begin{aligned}
S_{A C}^{\prime} S_{C B}^{\prime} & =S_{C A}^{\prime} S_{C B}^{\prime}=e_{C}^{\mu} \partial_{\mu} \phi_{A} e_{C}^{\nu} \partial_{\nu} \phi_{B}=g^{\mu \nu} \partial_{\mu} \phi_{A} \partial_{\nu} \phi_{B} \\
& =H_{A B} \equiv \eta_{A B}+\bar{h}_{A B},
\end{aligned}
$$

where the symmetry of $S_{A C}^{\prime}$ imposed by constraint (7) is used. This shows that the matrix $S_{A B}^{\prime}$ is the square root of the matrix $H_{A B}$ and, hence,

$$
\begin{aligned}
S_{A B}+\eta_{A B} & =\sqrt{\eta_{A B}+\bar{h}_{A B}} \\
& =\eta_{A B}+\frac{1}{2} \bar{h}_{A B}-\frac{1}{8} \bar{h}_{A}{ }^{C} \bar{h}_{C B}+\frac{1}{16} \bar{h}_{A}{ }^{C} \bar{h}_{C} D \bar{h}_{D B}+\cdots .
\end{aligned}
$$

Substituting this expansion in (9) one can easily check that the obtained Lagrangian will be reduced to (3) with Fierz-Pauli quadratic term (In the appendix we show how this comes 
out explicitly by solving perturbatively the equations for $\left.e_{A}^{\mu}\right)$. Moreover, the action for the scalar fields without higher order terms $O\left(S^{3}\right)$, that is,

$$
S_{\phi}=\frac{m_{g}^{2}}{8} \int d^{4} x \sqrt{-g}\left[S^{2}-S_{A B} S^{A B}\right]
$$

precisely corresponds to the theory [7], which is advocated to be ghost free [7, 8]. Now we have rewritten this theory in a form when the scalar fields comes only to second order. Therefore, if we can show that the scalar field $\phi^{0}$ becomes dynamical then there is no hope that the ghost can be canceled in higher orders via field redefinitions. Substituting

$$
e_{A}^{\mu}=\delta_{A}^{\mu}+l_{A}^{\mu}, \quad \phi^{A}=x^{A}+\chi^{A},
$$

in (6) we get

$$
S_{A B}=l_{A B}+\chi_{B, A}+l_{A}^{\mu} \chi_{B, \mu}
$$

in particular,

$$
S_{i 0}=l_{i 0}+\dot{\chi}_{i}+l_{i}^{0} \dot{\chi}_{0}+l_{i}^{k} \chi_{0, k},
$$

where dot denotes derivative with respect to time and we raise and lower all indices with the Minkowski metric. Keeping in action (16) only relevant terms and substituting there equation (19) we find

$$
S_{\phi}=\frac{m_{g}^{2}}{8} \int d^{4} x \sqrt{-g}\left[-S_{i 0} S^{i 0}+\ldots\right]=\frac{m_{g}^{2}}{8} \int d^{4} x \sqrt{-g}\left[l_{i}^{0} l_{i}^{0} \dot{\chi}_{0}^{2}+\ldots\right] .
$$

Notice that the fields $\chi^{A}$ are independent variables and $l_{A}^{\mu}$ take care about the constraints. If $l_{i}^{0} \neq 0$ the field $\chi^{0}$ propagates in a non-trivial background and there is no hope for this degree of freedom, even in principle, to be removed by a field redefinition because there are no higher order terms in the action which could cancel this mode (a perturbative expression for $l_{i}^{0}$ is given in the appendix).

Thus we find in a very straightforward manner that the ghost state associated with $\chi_{0}$ does not propagate except in a non-trivial background with non-vanishing $l_{i}^{0}$. However, on this background there is no way to avoid the ghost. This confirms our previous result [3] obtained through a long calculation, where the equations of motion were analyzed up to fourth order, and proves that the nonlinear BD ghost is unavoidable in massive gravity (see also [9]).

It is now possible to see that unlike the old non-Higgs like formulations of massive gravity, there is no problem here with the unacceptable constraint of vanishing linearized curvature [6]. The linearized form of equation (A.15) is given by

$$
\begin{aligned}
R_{\mu \nu} & =-\frac{m^{2}}{4}\left(S_{\mu \nu}-\eta_{\mu \nu} S\right) \\
& =-\frac{m^{2}}{4}\left(\partial_{\mu} \chi_{\nu}-\eta_{\mu \nu} \partial^{\rho} \chi_{\rho}\right)
\end{aligned}
$$


where $\partial_{\mu} \chi_{\nu}=\partial_{\nu} \chi_{\mu}$ by symmetry of $S_{\mu \nu}$. Thus the Bianchi identity implies

$$
\begin{aligned}
0 & =\partial^{\mu}\left(R_{\mu \nu}-\frac{1}{2} \eta_{\mu \nu} R\right) \\
& =-\frac{m^{2}}{4}\left(\partial^{2} \chi_{\nu}+\frac{1}{2} \partial_{\nu} \partial^{\rho} \chi_{\rho}\right) \\
& =-\frac{3 m^{2}}{8} \partial^{2} \chi_{\nu}
\end{aligned}
$$

which is the vanishing of the Laplacian of the scalar fields $\chi_{\nu}$. This is to be contrasted with the Pauli-Fierz formulation where the Bianchi identity implies the unacceptable condition $\partial^{\mu} h_{\mu \nu}=\partial_{\nu} h$ which is equivalent to the vanishing of linearized curvature.

We also like to mention that there exists a special combination of higher order terms as function of $S_{A B}$

$$
\int d^{4} x \sqrt{-g} \epsilon_{M N P Q} \epsilon^{A B C D} S_{A}^{M} S_{B}^{N} S_{C}^{P}\left(\frac{c_{1}}{3 !} \delta_{D}^{Q}+\frac{c_{2}}{4 !} S_{D}^{Q}\right)
$$

which could be thought of as generalization of the Fierz-Pauli form.

\section{A Perturbative expansion}

In this appendix we will solve the constraints explicitly using perturbation theory to check the consistency of the consideration above. Due to the constraints (5) and (6) the metric can be written as

$$
g^{A B}=\eta^{A B}+h^{A B}=\eta^{A B}+l^{A B}+l^{B A}+l_{C}^{A} l^{B C},
$$

while the symmetry of $S_{A B}$ gives

$$
\left(\delta_{A}^{\mu}+l_{A}^{\mu}\right)\left(\eta_{\mu B}+\partial_{\mu} \chi_{B}\right)-\left(\delta_{B}^{\mu}+l_{B}^{\mu}\right)\left(\eta_{\mu A}+\partial_{\mu} \chi_{A}\right)=0
$$

or equivalently

$$
l_{B A}-l_{A B}+\partial_{A} \chi_{B}-\partial_{B} \chi_{A}+l_{A}^{\mu} \partial_{\mu} \chi_{B}-l_{B}^{\mu} \partial_{\mu} \chi_{A}=0 .
$$

We now solve equations for the symmetric and antisymmetric parts of

$$
l_{A B}=p_{A B}+q_{A B},
$$

where $p_{A B}=p_{B A}$ and $q_{A B}=-q_{B A}$, perturbatively. With this purpose we write

$$
\begin{aligned}
& p_{A B}=p_{A B}^{(1)}+p_{A B}^{(2)}+p_{A B}^{(3)}+\cdots \\
& q_{A B}=q_{A B}^{(1)}+q_{A B}^{(2)}+q_{A B}^{(3)}+\cdots
\end{aligned}
$$


which being substituted in the constraint equations give

$$
\begin{aligned}
p_{A B}^{(1)} & =\frac{1}{2} h_{A B}, \\
q_{A B}^{(1)} & =\frac{1}{2}\left(\partial_{A} \chi_{B}-\partial_{B} \chi_{A}\right), \\
p_{A B}^{(2)} & =-\frac{1}{8}\left(h_{A C}+\partial_{A} \chi_{C}-\partial_{C} \chi_{A}\right)\left(h_{B}^{C}+\partial_{B} \chi^{C}-\partial^{C} \chi_{B}\right), \\
q_{A B}^{(2)} & =\frac{1}{4}\left(h_{C A}+\partial_{C} \chi_{A}-\partial_{A} \chi_{C}\right) \partial^{C} \chi_{B}-\frac{1}{4}\left(h_{C B}+\partial_{C} \chi_{B}-\partial_{B} \chi_{C}\right) \partial^{C} \chi_{A},
\end{aligned}
$$

Combining these relations we find

$$
\begin{aligned}
S_{A B} & =p_{A B}+\frac{1}{2}\left(\partial_{A} \chi_{B}+\partial_{B} \chi_{A}\right)+\frac{1}{2}\left(l_{A}^{\mu} \partial_{\mu} \chi_{B}+l_{B}^{\mu} \partial_{\mu} \chi_{A}\right) \\
& =\frac{1}{2} \bar{h}_{A B}-\frac{1}{8} \bar{h}_{A}{ }^{C} \bar{h}_{C B}+\cdots
\end{aligned}
$$

and one can continue this procedure to verify that the formal result (15) holds.

Having seen that the fields $e_{A}^{\mu}$ depend on the fields $g^{\mu \nu}$ and $\partial_{\mu} \phi_{A}$ through relations that could be only solved perturbatively, it is essential to impose the constraints through Lagrange multipliers to get the correct equations of motion. First the $g^{\mu \nu}$ equation of motion gives

$$
R_{\mu \nu}-\frac{1}{2} g_{\mu \nu} R=\tau_{\mu \nu}-\frac{1}{2} g_{\mu \nu} \tau
$$

and thus

$$
\tau_{\mu \nu}=R_{\mu \nu}
$$

The $e_{A}^{\mu}$ equations give

$$
\lambda^{A B} \partial_{\mu} \phi_{B}=R_{\mu \nu} e^{\nu A}+\frac{m^{2}}{4}\left(S^{A B} \partial_{\mu} \phi_{B}-S \partial_{\mu} \phi^{A}\right),
$$

which is a set of 16 equations, ten for the Ricci tensor $R_{\mu \nu}$ and six for the antisymmetric matrices $\lambda^{A B}$. The $\phi^{A}$ equations of motion give

$$
\partial_{\mu}\left(\sqrt{-g} \lambda^{A B} e_{A}^{\mu}\right)=\frac{m^{2}}{4} \partial_{\mu}\left(\sqrt{-g}\left(S^{A B} e_{A}^{\mu}-S e^{\mu B}\right)\right) .
$$

Assuming that the "vierbein" $\partial_{\mu} \phi_{B}=\eta_{\mu B}+\partial_{\mu} \chi_{B}$ has an inverse, we deduce that

$$
\begin{aligned}
\lambda^{A B} & =\frac{1}{2} R_{\mu \nu}\left(e^{\nu A}\left(\partial_{\mu} \phi_{B}\right)^{-1}-e^{\nu B}\left(\partial_{\mu} \phi_{A}\right)^{-1}\right), \\
-\frac{m^{2}}{4}\left(S^{A B}-\eta^{A B} S\right) & =\frac{1}{2} R_{\mu \nu}\left(e^{\nu A}\left(\partial_{\mu} \phi_{B}\right)^{-1}+e^{\nu B}\left(\partial_{\mu} \phi_{A}\right)^{-1}\right) .
\end{aligned}
$$

Notice that because of the symmetry of $R_{\mu \nu}$ the first non-vanishing contribution to $\lambda^{A B}$ is of order two as can be seen by using a perturbative expansion of $\left(\partial_{\mu} \phi_{B}\right)^{-1}$.

\section{Acknowledgments}

A. H. C is supported in part by the National Science Foundation under Grant No. Phys0854779. V.M. is supported by TRR 33 "The Dark Universe" and the Cluster of Excellence EXC 153 "Origin and Structure of the Universe." 
Open Access. This article is distributed under the terms of the Creative Commons Attribution Noncommercial License which permits any noncommercial use, distribution, and reproduction in any medium, provided the original author(s) and source are credited.

\section{References}

[1] A.H. Chamseddine and V. Mukhanov, Higgs for graviton: simple and elegant solution, JHEP 08 (2010) 011 [arXiv: 1002.3877] [SPIRES].

[2] L. Alberte, A. Chamseddine and V. Mukhanov, Massive gravity: resolving the puzzles, JHEP 12 (2010) 023 [arXiv: 1008.5132] [SPIRES].

[3] L. Alberte, A.H. Chamseddine and V. Mukhanov, Massive gravity: exorcising the ghost, JHEP 04 (2011) 004 [arXiv: 1011.0183] [SPIRES].

[4] K. Hinterbichler, Theoretical aspects of massive gravity, arXiv:1105.3735 [SPIRES].

[5] M. Fierz and W. Pauli, On relativistic wave equations for particles of arbitrary spin in an electromagnetic field, Proc. Roy. Soc. Lond. A 173 (1939) 211 [SPIRES].

[6] D.G. Boulware and S. Deser, Can gravitation have a finite range?, Phys. Rev. D 6 (1972) 3368 [SPIRES].

[7] C. de Rham, G. Gabadadze and A.J. Tolley, Resummation of massive gravity, Phys. Rev. Lett. 106 (2011) 231101 [arXiv:1011.1232] [SPIRES].

[8] S.F. Hassan and R.A. Rosen, Resolving the ghost problem in non-linear massive gravity, arXiv: 1106.3344 [SPIRES].

[9] S. Folkerts, A. Pritzel and N. Wintergerst, On ghosts in theories of self-interacting massive spin-2 particles, arXiv:1107.3157 [LMU-ASC-30-11] [SPIRES]. 